\title{
Transforming growth factor- $\beta 1$ induces type II collagen and aggrecan expression via activation of extracellular signal-regulated kinase 1/2 and Smad2/3 signaling pathways
}

\author{
YANHUI ZHU, HAIRONG TAO, CHEN JIN, YONZHANG LIU, XIONGWEI LU, \\ XIAOPENG HU and XIANG WANG \\ Department of Orthopaedics, Shanghai Ninth People's Hospital, Shanghai Jiao Tong University School of Medicine, \\ Shanghai 201900, P.R. China
}

Received June 1, 2014; Accepted June 17, 2015

DOI: $10.3892 / \mathrm{mmr} .2015 .4068$

\begin{abstract}
Transforming growth factor (TGF) $-\beta$ regulates the anabolic metabolism of articular cartilage and prevents cartilage degradation. TGF- $\beta 1$ influences cellular proliferation, differentiation and the extracellular matrix through activation of the extracellular signal-regulated kinase (ERK) $1 / 2$ and Smad2/3 signaling pathways. However, it has remained to be fully elucidated precisely how the ERK1/2 and Smad2/3 signaling pathways mediate anabolic processes of articular cartilage. The present study investigated how ERK1/2 and Smad2/3 signaling mediate TGF- $\beta 1$-stimulated type II collagen and aggrecan expression in rat chondrocytes. The results confirmed that TGF- $\beta 1$ stimulates type II collagen and aggrecan expression in rat chondrocytes, and furthermore, that the ERK1/2 and Smad2/3 signaling pathways were activated by TGF- $\beta 1$. Conversely, the TGF- $\beta$ receptor I (ALK5) kinase inhibitor SB525334 significantly impaired TGF- $\beta 1$-induced type II collagen and aggrecan expression, coinciding with a reduction of ERK1/2 and Smad3 phosphorylation. In addition, TGF- $\beta 1$-induced type II collagen and aggrecan expression were significantly suppressed by ERK1/2 inhibitor PD98059. Similarly, TGF- $\beta 1$-stimulated type II collagen and aggrecan expression were decreased in the presence of a Smad3 phosphorylation inhibitor SIS3. Therefore, the present study demonstrated that the ERK1/2 and Smad2/3 signaling pathways regulate type II collagen and aggrecan expression in rat chondrocytes.
\end{abstract}

Correspondence to: Mr. Xiang Wang, Department of Orthopaedics, Shanghai Ninth People's Hospital, Shanghai Jiao Tong University School of Medicine, 280 Mohe Road, Shanghai 201900, P.R. China E-mail:wx20022005@outlook.com

Key words: transforming growth factor beta 1, type II collagen, aggrecan, extracellular-regulated kinase $1 / 2$, Smad, chondrocytes

\section{Introduction}

The progressive loss of extracellular matrix (ECM) in cartilage is the characteristic lesion in osteoarthritis (OA). Type II collagen and aggrecan are the main components of cartilage ECM, and decreased production of either may irreversibly lead to cartilage degradation. Transforming growth factor- $\beta$ (TGF- $\beta$ ) is an important regulator of the anabolic metabolism of articular cartilage. TGF- $\beta$ enhances ECM production by increasing synthesis of type II collagen and aggrecan, thereby preventing cartilage degradation $(1,2)$. Reduced type II collagen and aggrecan expression is a characteristic of aged, osteoarthritic and passaged chondrocytes. However, TGF- $\beta$ can stimulate expression of type II collagen and aggrecan in these cells (3). Furthermore, bone-marrow mesenchymal stem cells exposed to TGF- $\beta$ express chondrogenic markers (type II collagen and aggrecan), implying their differentiation into chondrocytes $(4,5)$. In addition, levels of type II collagen and aggrecan in bovine intervertebral disc cells increase following TGF- $\beta 1$ exposure, suggesting that it can also act in the prevention of degenerative disc disease (6).

TGF- $\beta$ activates the Smad2/3 signaling pathway, stimulating expression of type II collagen $\alpha 1$, aggrecan and sex determining region Y-box 9 expression in human $\mathrm{OA}$ and bovine chondrocytes (7). TGF- $\beta$ can also activate extracellular signal-regulated kinase (ERK)1/2 to regulate anabolic activity through upregulation of TIMP-3 expression and chondrocyte cell growth $(8,9)$. Furthermore, TGF- $\beta 1$ can activate Smad2 and ERK1/2 signaling pathways in human mesenchymal stem cells, subsequently increasing the expression of type II collagen and aggrecan (10). Only a few studies have described the role of ERK1/2 and Smad2/3 signaling pathways in TGF- $\beta 1$-induced type II collagen and aggrecan expression in rat chondrocytes $(8,11)$.

The present study investigated the effect of TGF- $\beta 1$ on type II collagen and aggrecan expression in rat chondrocytes as well as the possible mechanistic involvement of ERK1/2 and Smad2/3 signaling pathways. For this, either ERK1/2 or Smad2/3 signaling pathways were blocked with small molecules, and the effect on TGF- $\beta 1$-induced type II collagen and aggrecan expression was investigated. A previous study by our 
group suggested that inhibition of ERK1/2 signaling may be used for the pharmacological intervention of cartilage breakdown in OA (12). In addition to the inhibition of the ERK1/2 signaling pathway, subsequent suppression of TGF- $\beta 1$-induced anabolic activity should be considered according to the results of the present study.

\section{Materials and methods}

Chondrocyte isolation and culture. A total of 20 female Sprague-Dawley rats (weight, 180-200 g) were supplied by the Animal Center, Shanghai Jiao Tong University School of Medicine (Shanghai, China). The rats were sacrificed by cervical dislocation prior to surgical removal of normal rat knee cartilage from the tibial platform and the femoral condyle. The experiments were conducted with approval from the Shanghai Jiao Tong University School of Medicine ethics committee. Cartilage pieces were washed with phosphate-buffered saline (PBS) twice and treated with $0.2 \mathrm{mg} / \mathrm{ml}$ collagenase (Sigma-Aldrich, St. Louis, MO, USA) in serum-free Dulbecco's modified Eagle's medium (DMEM; Gibco Life Technologies, Carlsbad, CA, USA) overnight at $37^{\circ} \mathrm{C}$. The cells were collected by filtering the disaggregated tissue through a 200-mesh nylon cell strainer, centrifugation at $1,000 \mathrm{x}$ f for $5 \mathrm{~min}$ and two washes with PBS. Finally, the cells were re-suspended and cultured in DMEM supplemented with $10 \%$ (vol/vol) fetal bovine serum (Invitrogen Life Technologies, Carlsbad, CA, USA), plus $1 \%$ penicillin and streptomycin (Gibco Life Technologies). The culture medium was replaced every second day. The chondrocytic phenotype of the cultured cells was confirmed by positive immunostaining for type II collagen. Briefly, cells cultured on glass slides were incubated overnight at $4^{\circ} \mathrm{C}$ with anti-rat type II collagen (1:300 dilution; cat. no. ab34712; Abcam, Cambridge, UK), and immunostaining was performed using immunostaining reagent (Dolichos bifows agglutini Immunohistochemistry kit; Wuhan Boster Biological Technology, Ltd., Wuhan, China), according to the manufacturer's instructions. Positive immunostaining of collagen II was detected using an inverted fluorescence microscope (IX70; Olympus, Tokyo, Japan). Toluidine blue staining (Shanghai Chemical Reagent Co., Ltd., Shanghai, China) of glycosaminoglycans was also conducted. First-passage chondrocytes were used in all experiments.

Chondrocyte treatment. First-passage chondrocytes were trypsinized and seeded into six-well plates $\left(3 \times 10^{5}\right.$ cells/well) for $24 \mathrm{~h}$. Chondrocytes were maintained in serum-deficient DMEM for $24 \mathrm{~h}$ and subjected to a variety of treatments. Chondrocytes were cultured with or without $10 \mu \mathrm{g} / \mathrm{ml} \mathrm{recom-}$ binant human TGF- $\beta 1$ (Perprotech, Rocky Hill, NJ, USA) for $48 \mathrm{~h}$. Type II collagen and aggrecan expression was evaluated by reverse transcription quantitative polymerase chain reaction (RT-qPCR) and western blotting. Chondrocytes were cultured with $10 \mu \mathrm{g} / \mathrm{ml} \mathrm{TGF- \beta 1.} \mathrm{Following} 0,1,5,10,15,30,60$ and $180 \mathrm{~min}$ of incubation, phosphorylated (p)-Smad3, Smad2/3, p-ERK1/2, and ERK1/2 levels were examined by western blotting. For inhibition studies, chondrocytes were pre-treated in serum-free medium with or without TGF- $\beta$ receptor I (ALK5) kinase inhibitor SB525334 (1 $\mu \mathrm{M}$; Selleckchem, Houston, TX, USA), the pharmacological ERK1/2 inhibitor
PD98059 (10 $\mu \mathrm{M}$; Promega Corporation, Madison, WI, USA), and pharmacological Smad3 phosphorylation inhibitor SIS3 (10 $\mu \mathrm{M}$; Santa Cruz Biotechnology, Inc. Dallas, TX, USA) for $60 \mathrm{~min}$ and then stimulated with or without $10 \mu \mathrm{g} / \mathrm{ml} \mathrm{TGF}-\beta 1$ for $48 \mathrm{~h}$. Expression of type II collagen and aggrecan was evaluated by RT-qPCR and western blotting. To examine the involvement of the ERK1/2 and Smad signaling pathways, chondrocytes were pre-treated in serum-free medium with SB525334, PD98059 or SIS3 for $60 \mathrm{~min}$, followed by stimulation with $10 \mu \mathrm{g} / \mathrm{ml}$ TGF- $\beta 1$. Following $0,1,5,10,15,30,60$ or $180 \mathrm{~min}$ of incubation, p-Smad3, Smad2/3, p-ERK1/2, and ERK1/2 levels were examined by western blotting.

$R N A$ extraction and $R T-q P C R$. Total RNA was extracted from chondrocytes using TRIzol reagent (Invitrogen Life Technologies) according to the manufacturer's instructions. For first-strand cDNA synthesis, $2 \mu \mathrm{g}$ mRNA was reverse-transcribed using the Reverse Transcriptase Moloney murine leukemia virus (M-MLV) cDNA Synthesis kit (Takara, Tokyo, Japan) according to the manufacturer's instructions. Briefly, a $12-\mu 1$ reaction mixture containing $2 \mu \mathrm{l}$ oligo $\mathrm{d}(\mathrm{T}) 18$ primer $(50 \mu \mathrm{M}), 2 \mu \mathrm{g}$ total RNA and RNase-free $\mathrm{dH}_{2} \mathrm{O}$ was incubated at $70^{\circ} \mathrm{C}$ for $10 \mathrm{~min}$. Subsequently, $1 \mu \mathrm{l}$ deoxynucleotide triphosphate mixture (10 mM each), $4 \mu 15 \mathrm{X}$ M-MLV buffer, $0.5 \mu 1$ ribonuclease inhibitor $(40 \mathrm{U} / \mu \mathrm{l}), 1 \mu \mathrm{l}$ reverse transcriptase M-MLV (RNase H-free; $200 \mathrm{U} / \mu \mathrm{l}$ ) and RNase-free $\mathrm{dH}_{2} \mathrm{O}$ were added to a final volume of $20 \mu \mathrm{l}$ and the mixture was incubated for $60 \mathrm{~min}$ at $42^{\circ} \mathrm{C}$. Next, the reaction was inactivated by heating at $70^{\circ} \mathrm{C}$ for $15 \mathrm{~min}$. Subsequently, $2 \mathrm{ml}$ of the $20 \mathrm{ml}$ reverse transcription reaction was subjected to PCR amplification using PCR Master Mix (cat. no. M7502, Promega Corporation) with initial denaturation at $95^{\circ} \mathrm{C}$ for $10 \mathrm{~min}$, then 35 cycles of $94^{\circ} \mathrm{C}$ for $30 \mathrm{sec}, 60^{\circ} \mathrm{C}$ for $30 \mathrm{sec}$ and $72^{\circ} \mathrm{C}$ for $45 \mathrm{sec}$. Products were quantified using a melting curve analysis. Amplification of GAPDH was performed to quantify PCR products and confirm the use of equal amounts of RNA. Relative gene expression was analyzed using the $2^{\Delta \Delta \mathrm{Ct}}$ method (13). Primer sequences (Shanghai Sangon Biotechnology Co., Ltd., Shanghai, China) of rat genes were as follows: Type II collagen forward, 5'-TCCTAAGGGTGCCAATGGTGA-3', and reverse, 5'-GGACCAACTTTGCCTTGAGGAC-3'; aggrecan forward, 5'-TCCGCTGGTCTGATGGACAC-3', and reverse, 5'-CCAGATCATCACTACGCAGTCCTC-3'; and GAPDH forward, 5'-CAAGTTCAACGGCACAGTCAAG-3', and reverse, 5'-ACATACTCAGCACCAGCATCAC-3'.

Protein extraction and western blot analysis. Whole-cell lysates were prepared using radioimmunoprecipitation assay buffer [50 mM Tris- $\mathrm{HCl}$ ( $\mathrm{pH} 7.4), 150 \mathrm{mM} \mathrm{NaCl}$, $1 \%$ Triton $\mathrm{X}-100,0.1 \% \mathrm{SDS}, 2 \mathrm{mM}$ EDTA and $2 \mathrm{mM}$ phenylmethanesulfonylfluoride] in the presence of protease and phosphatase inhibitors. For the detection of p-Smad3, Smad2/3, p-ERK1/2, ERK1/2 and aggrecan protein levels, equal amounts of total cellular extracts from rat chondrocytes were then loaded on a $10 \%$ SDS-polyacrylamide gel, subjected to electrophoresis and transferred to polyvinylidene fluoride membranes (EMD Millipore, Billerica, MA, USA) by electroblotting. For type II collagen protein levels, equal amounts of total cellular extracts were separated by $8 \%$ SDS-polyacrylamide gel electrophoresis. Membranes 
A

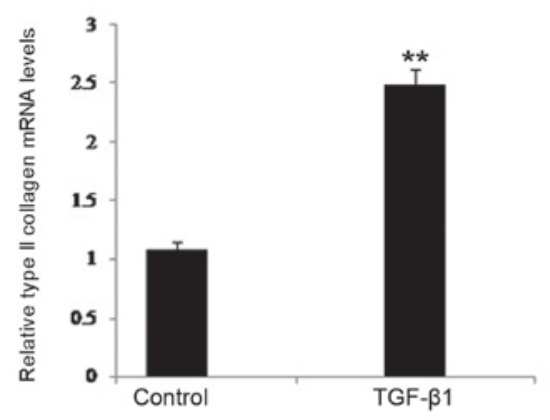

B

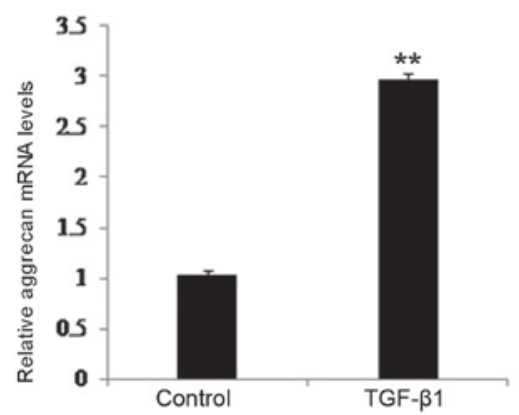

D
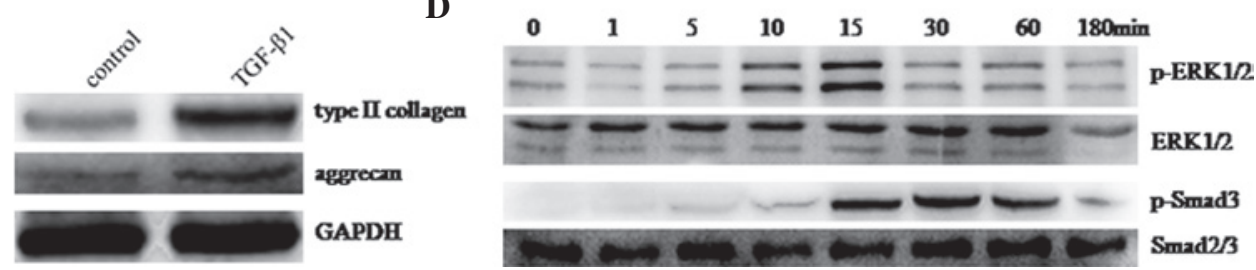

Figure 1. TGF- $\beta 1$-stimulated type II collagen and aggrecan expression is mediated via ERK1/2 and Smad2/3 signaling pathways. (A and B) Type II collagen and aggrecan mRNA levels. Values are expressed as the mean \pm standard deviation. ${ }^{* *} \mathrm{P}<0.01$ vs. control. (C) Type II collagen and aggrecan protein levels as evaluated by western blot analysis. (D) At the indicated time-points, ERK1/2, p-ERK1/2, p-Smad3, and Smad2/3 levels were assessed by western blotting. The blots were stripped and re-probed with regular antibodies to detect the total amount of the respective proteins. p-ERK, phosphorylated extracellular signal-regulated kinase; TGF, transforming growth factor.

were then blocked with 5\% skimmed milk. Polyclonal anti-rat type II collagen (1:5,000 dilution; cat. no. ab34712; Abcam), polyclonal anti-rat aggrecan (1:200 dilution; cat. no. 251591; Abbiotec, San Diego, CA, USA), monoclonal anti-rat p-Smad3 (1:1,000 dilution; cat. no. ab52903; Abcam), polyclonal anti-rat Smad2/3 (1:500 dilution; cat. no. R-1002-100; Novus, Littleton, CO, USA), monoclonal anti-rat p-ERK1/2 (1:1,000 dilution; cat. no. ab32538; Abcam) and polyclonal anti-rat ERK1/2 antibody (1:1,000; cat. no. 9102; Cell Signaling Technology Inc., Danvers, MA, USA) were incubated with the membranes overnight at $4^{\circ} \mathrm{C}$. The membranes were then washed and reacted with secondary horseradish peroxidase-conjugated secondary antibody [donkey anti-rabbit immunoglobulin $\mathrm{G}$ for type II collagen, aggrecan, p-Smad3, Smad2/3, p-ERK1/2, and ERK1/2 (1:2,000; cat. no. sc-2305; Santa Cruz Biotechnology, Inc.)] at room temperature for $2 \mathrm{~h}$. Finally, protein bands were visualized by chemiluminescence (EMD Millipore) using the Tanon 4200 SF Imaging Analysis system (Tanon Science and Technology Co., Ltd., Shanghai, China). Western blots were re-probed with a monoclonal anti-GAPDH antibody (1:1,000 dilution; cat. no. 2118; Cell Signaling Technology Inc.) as a control.

Statistical analysis. All experiments were performed three times and the results are expressed as the mean \pm standard deviation. Statistical significance was determined using the Mann-Whitney U test or Kruskal-Wallis analysis of variance test, when appropriate, using SPSS 13.0 statistical software (SPSS, Inc., Chicago, IL, USA). $\mathrm{P}<0.05$ was considered to indicate a statistically significant difference between values.

\section{Results}

ERK1/2 and Smad2/3 signaling pathways mediate TGF- $\beta 1$-stimulated type II collagen and aggrecan expression in rat chondrocytes. To confirm that TGF- $\beta 1$ induced upregulation of type II collagen and aggrecan expression in chondrocytes, rat chondrocytes were cultured with or without TGF- $\beta 1$ for $48 \mathrm{~h}$. RT-qPCR and western blotting were then performed to examine the expression of type II collagen and aggrecan. As shown in Fig. 1A and B, significant upregulation of type II collagen and aggrecan occurred following treatment with TGF- $\beta 1$. Western blotting also indicated that type II collagen and aggrecan protein levels were increased following TGF- $\beta 1$ treatment (Fig. $1 \mathrm{C}$ ). These results confirmed that TGF- $\beta 1$ stimulated type II collagen and aggrecan expression in rat chondrocytes.

Furthermore, it was evaluated whether the ERK1/2 and Smad2/3 signaling pathways were involved in TGF- $\beta 1$ stimulation of type II collagen and aggrecan. Rat chondrocytes were cultured in the presence of TGF- $\beta 1$, and at the indicated time-points, the activation of ERK1/2 and phosphorylation of Smad3 were examined by western blotting. Fig. 1D demonstrates that TGF- $\beta 1$ induced rapid phosphorylation of ERK1/2 and Smad3. These observations suggested that the ERK1/2 and Smad2/3 signaling pathways may have an important role in TGF- $\beta 1$-stimulated type II collagen and aggrecan induction in rat chondrocytes.

Blockade of TGF- $\beta 1$ receptor represses the stimulation of type II collagen and aggrecan expression. To further investigate the involvement of the ERK1/2 and Smad2/3 signaling pathways in TGF- $\beta 1$-induced type II collagen and aggrecan expression, chondrocytes were pre-treated with or without ALK5 kinase inhibitor SB525334 and then stimulated with or without TGF- $\beta 1$. After $48 \mathrm{~h}$, RT-qPCR and western blotting confirmed that TGF- $\beta 1$ stimulated type II collagen and aggrecan expression was inhibited by SB525334 (Fig. 2A-C). At the indicated time-points, western blotting demonstrated that phosphorylation of ERK1/2 and Smad3 was blocked by SB525334 (Fig. 2D). These results further validated the role of the ERK1/2 and Smad2/3 signaling pathways in type II collagen and aggrecan induction by TGF- $\beta 1$. 
A

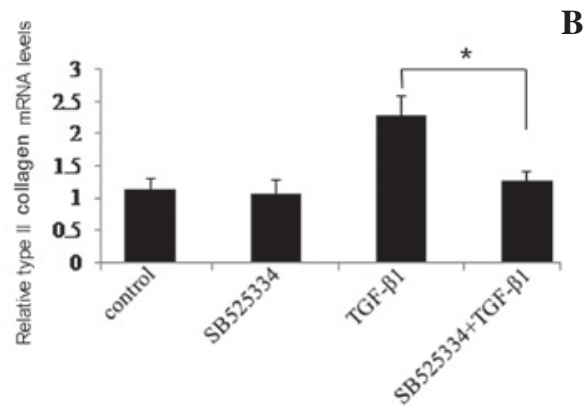

B

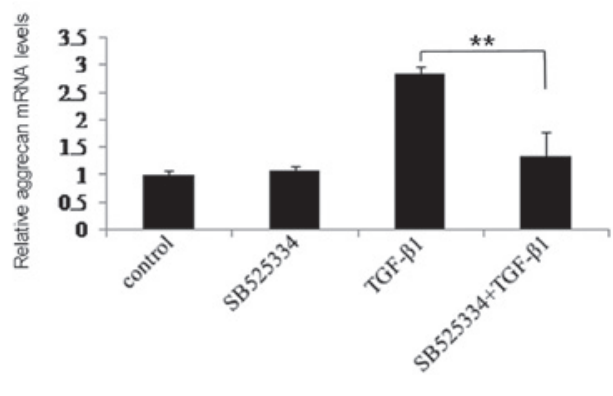

C
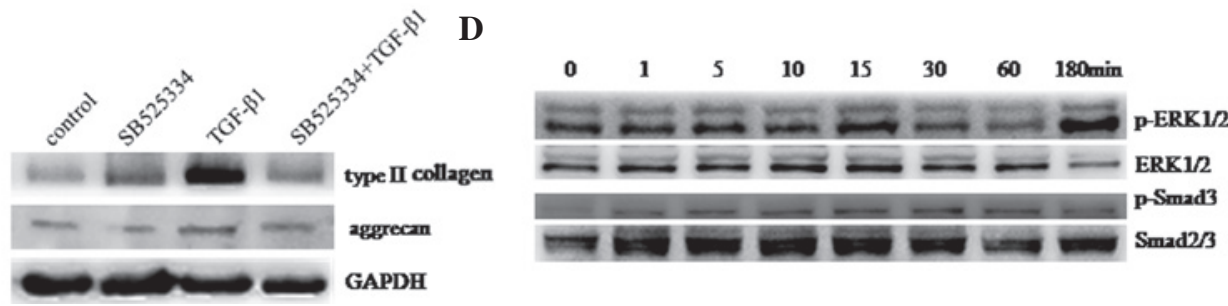

Figure 2. Inhibition of ERK1/2 and Smad2/3 signaling prevents TGF- $\beta 1$-stimulated type II collagen and aggrecan expression. (A and B) Type II collagen and aggrecan mRNA levels. Values are expressed as the mean \pm standard deviation. ${ }^{*} \mathrm{P}<0.01 ;{ }^{* *} \mathrm{P}<0.01$. (C) Type II collagen and aggrecan protein levels as evaluated by western blot analysis. (D) Chondrocytes were treated with TGF- $\beta 1$ following pre-treatment with TGF- $\beta$ receptor I kinase inhibitor SB525334. At the indicated time-points, ERK1/2, p-ERK1/2, p-Smad3, and Smad2/3 levels were determined by western blotting. The blots were stripped and re-probed with regular antibodies to detect the total amount of the respective proteins. p-ERK, phosphorylated extracellular signal-regulated kinase; TGF, transforming growth factor.

A

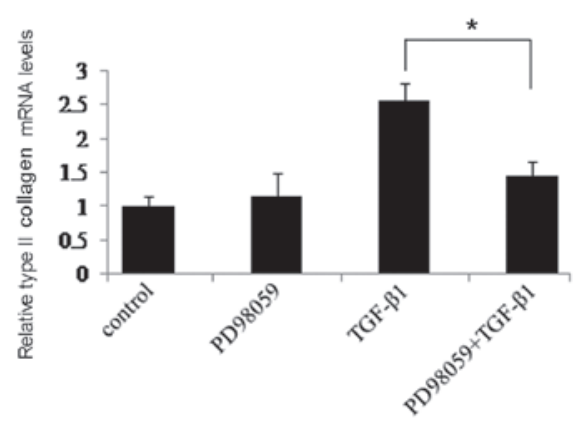

B

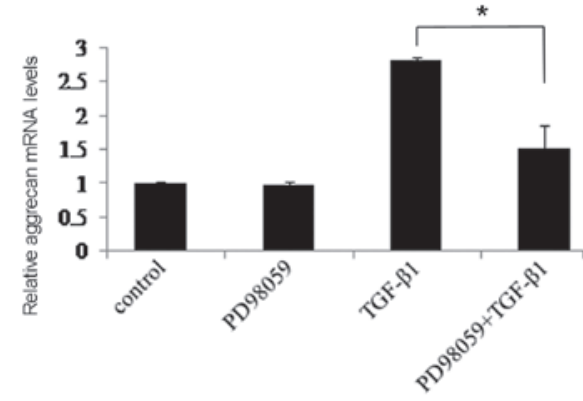

C

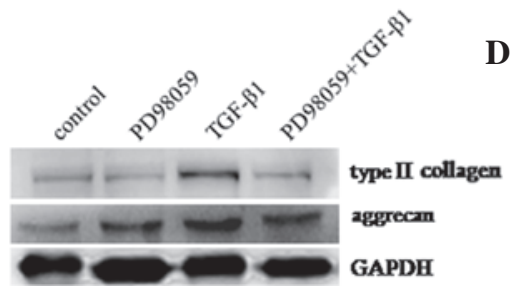

D

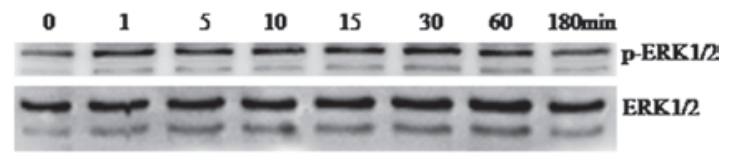

Figure 3. Blockade of TGF- $\beta 1$-stimulated type II collagen and aggrecan expression by inhibition of the ERK1/2 pathway. (A and B) Type II collagen and aggrecan mRNA levels. Values are expressed as the mean \pm standard deviation. ${ }^{*} \mathrm{P}<0.05$. (C) Type II collagen and aggrecan protein levels as evaluated by western blot analysis. (D) Chondrocytes were treated with TGF- $\beta 1$ following pre-treatment with ERK1/2 inhibitor PD98059. At the indicated time-points, p-ERK1/2 and ERK1/2 levels were measured by western blotting. The blots were stripped and re-probed with regular antibodies to detect the total amount of the respective proteins. p-ERK, phosphorylated extracellular signal-regulated kinase; TGF, transforming growth factor.

Blockade of TGF- $\beta 1$-stimulated type II collagen and aggrecan expression by inhibition of ERK1/2 signaling. Next, the the role of ERK1/2 in type II collagen and aggrecan induction by TGF- $\beta 1$ was examined. Chondrocytes were pre-treated with or without ERK1/2 inhibitor PD98059 followed by culture with or without TGF- $\beta 1$ for $48 \mathrm{~h}$. RT-qPCR and western blotting revealed that TGF- $\beta 1$-induced expression of type II collagen and aggrecan was significantly suppressed in the presence of PD98059 (Fig. 3A-C). ERK1/2 signaling pathway activation was then assessed in chondrocytes pre-treated with PD98059 followed by culture with TGF- $\beta 1$. Activation of the ERK1/2 signaling pathway was inhibited by PD98059 (Fig. 3D). These results suggested that the ERK1/2 signaling pathway may have an important role in type II collagen and aggrecan induction by TGF- $\beta 1$.

Blockade of TGF- $\beta 1$-stimulated type II collagen and aggrecan expression by inhibition of $p$-Smad3. To determine whether the Smad2/3 signaling pathway affected TGF- $\beta 1$-induced type II collagen and aggrecan, SIS3 was used to suppress Smad3 phosphorylation. First, chondrocytes were cultured with or without TGF- $\beta 1$ for $48 \mathrm{~h}$ following pre-treatment with 
A

A

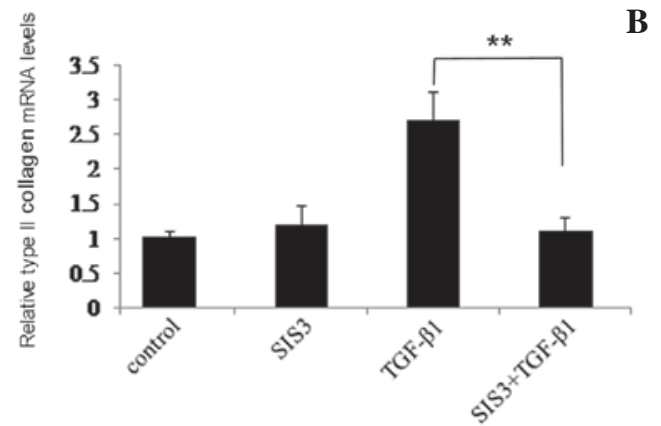

B

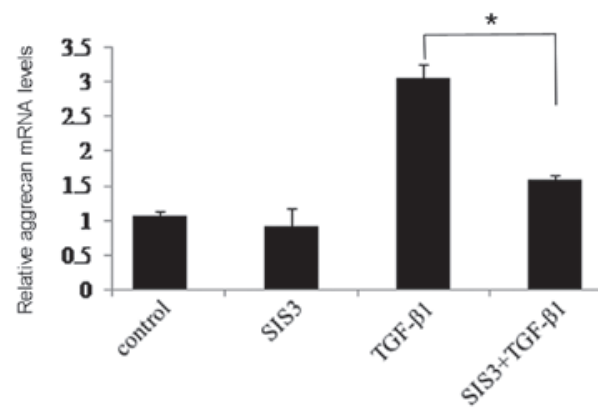

C

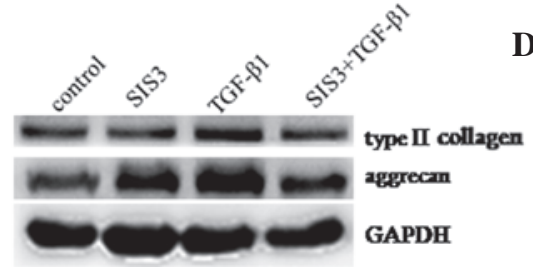

D

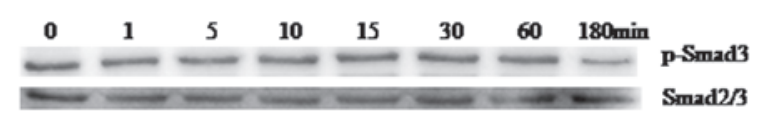

Figure 4. Blockade of TGF- $\beta 1$-stimulated type II collagen and aggrecan expression by inhibition of p-Smad3. (A and B) Type II collagen and aggrecan mRNA levels. Values are expressed as the mean \pm standard deviation. $\mathrm{P}<0.05,{ }^{* *} \mathrm{P}<0.01$. (C) Type II collagen and aggrecan protein levels as evaluated by western blot analysis. (D) Chondrocytes were treated with TGF- $\beta 1$ following pre-treatment with Smad3 phosphorylation inhibitor SIS3. At the indicated time-points, p-Smad3 and Smad2/3 levels were assessed by western blotting. The blots were stripped and re-probed with regular antibodies to detect the total amount of the respective proteins. TGF, transforming growth factor; $\mathrm{p}$, phosphorylated.

or without SIS3. RT-qPCR and western blotting indicated that TGF- $\beta 1$-induced type II collagen and aggrecan expression were significantly inhibited by SIS3 (Fig. 4A-C). Next, chondrocytes were treated with TGF- $\beta 1$ following pre-treatment with SIS3. At the indicated time-points, western blotting showed that Smad3 phosphorylation was markedly suppressed by SIS3 (Fig. 4C). These results suggested that the Smad2/3 signaling pathway may also have an important role in type II collagen and aggrecan induction by TGF- $\beta 1$.

\section{Discussion}

TGF- $\beta$ exerts profound effects on cellular proliferation, differentiation and modulation of ECM, regulating the anabolic process of articular cartilage and preventing cartilage degradation. It can stimulate full-depth explants of human OA knee articular cartilage to expression of type II collagen and aggrecan (1). A study found that adenoviral vector-mediated gene transduction of TGF- $\beta 1$ increased type II collagen and aggrecan expression in normal and osteoarthritic human chondrocytes (2). In addition, in bovine articular chondrocytes, transgene-mediated TGF- $\beta$ overexpression increased type II collagen and aggrecan expression (14). In analogy with this the present study showed that TGF- $\beta 1$ significantly induced type II collagen and aggrecan expression in rat chondrocytes. These results suggested that TGF- $\beta 1$ regulates the anabolic metabolism of articular cartilage and prevents cartilage degradation.

TGF- $\beta 1$ signals are transmitted through phosphorylation of the Smad2/3 by activation of type I (TGF- $\beta$ RI) and type II (TGF- $\beta$ RII) receptors (15-17). Phosphorylated Smad 2 and Smad3 form hetero-oligomeric complexes with Smad4 and migrate to the nucleus, where they regulate the transcription of target genes (17-19). TGF- $\beta$ also activates ERK1/2 to regulate the expression of target genes and cellular proliferation (20-23). Either or both the ERK1/2 or Smad2/3 signaling pathways are involved in TGF- $\beta 1$ sub-family-induced type II collagen gene expression (24-26). The results of the present study revealed that TGF- $\beta 1$ rapidly induced phosphorylation of ERK1/2 and Smad3, suggesting that activation of ERK1/2 as well as Smad2/3 signaling may account for TGF- $\beta 1$-induced type II collagen and aggrecan expression. Furthermore, the present study demonstrated that the ALK5 inhibitor SB525334 significantly impaired TGF- $\beta 1$-induced type II collagen and aggrecan expression. In addition, SB525334 suppressed TGF- $\beta 1$-stimulated phosphorylation of ERK1/2 and Smad3. In conclusion, these results strongly implicated ERK1/2 and Smad2/3 signaling in TGF- $\beta 1$-induced type II collagen and aggrecan expression.

Additional evidence of a role for ERK1/2 signaling in TGF- $\beta 1$-induced type II collagen and aggrecan expression was provided by the finding that PD98059 significantly inhibited TGF- $\beta 1$-induced type II collagen and aggrecan expression. Furthermore, TGF- $\beta 1$-stimulated phosphorylation of ERK1/2 was inhibited by PD98059. This indicated that activation of ERK1/2 signaling is an essential mechanism by which TGF- $\beta 1$ induces type II collagen and aggrecan expression. Therefore, inhibition of ERK1/2 signaling may impair TGF- $\beta 1$-stimulated anabolic metabolism of articular cartilage. The present study also investigated inhibition of Smad2/3 signaling pathway in TGF- $\beta 1$-induced type II collagen and aggrecan expression in rat chondrocytes using SIS3, a specific inhibitor of Smad3 phosphorylation. The results showed that TGF- $\beta 1$-induced type II collagen and aggrecan expression was significantly impaired by SIS3. Furthermore, TGF- $\beta 1$-stimulated phosphorylation of Smad3 was suppressed by SIS3. These results further suggested a crucial role for Smad2/3 signaling in the regulation of TGF- $\beta 1$-induced type II collagen and aggrecan expression.

The results of the present study strongly implicated the ERK1/2 and Smad2/3 signaling pathways in TGF- $\beta 1$-induced type II collagen and aggrecan expression. The results 
provided a functional link between ECM proteins, TGF- $\beta 1$ activity and disease, suggesting novel therapeutic opportunities for intervention in osteoarthritis. The ERK1/2 signaling pathway transduces signals from the cell membrane to the nucleus in response to a variety of stimuli, controlling a wide spectrum of cellular processes, including growth, differentiation and the expression of multiple genes. It is well documented that interleukin (IL)-1 $\beta$ is an important mediator of cartilage destruction during the OA process. IL-1 $\beta$ selectively induces ERK1/2 activation and stimulates the production of matrix metalloproteinases and aggrecanases $(27,28)$, which may contribute to cartilage loss and induce degradation of cartilage. This suggests that ERK1/2 signaling may have an important role in IL-1-induced degradation of cartilage. A previous study by our group showed such a requirement of ERK1/2 pathways for IL- $\beta$ inhibition of type II collagen and aggrecan in human chondrocytes (12). Therefore, ERK1/2 represents a potential therapeutic drug target for OA.

PD98059 prevents cyclosporine A-induced nuclear translocation of Smad2/3 and apoptosis in renal proximal tubular cells, suggesting the existence of crosstalk between ERK1/2 and Smad2/3 (29). ERK1/2 is also capable of phosphorylating Smads (30). Based on the results of the present study, exploiting ERK1/2 as a therapeutic drug target for OA may lead to decreased TGF- $\beta 1$-induced anabolic metabolism or impaired repair responses in chondrocytes through inhibition of ERK1/2 and indirect inhibition of Smad2/3. Although TGF- $\beta 1$ and IL- $\beta$ have opposing effects on type II collagen and aggrecan expression, they exert their effects through the same signaling pathway, possibly by activating different downstream transcription factors. Further studies are required to examine the downstream transcription factors influenced by ERK1/2 activation by various stimuli.

In conclusion, the present study showed that the ERK1/2 and Smad2/3 signaling pathways are required for the induction of type II collagen and aggrecan expression by TGF- $\beta 1$. This regulation of type II collagen and aggrecan expression may be pivotal in TGF- $\beta$-induced ECM protein synthesis and maintenance, and in the cartilage repair response. In addition, although inhibition of ERK1/2 signaling represents a valuable potential therapeutic drug target for OA (12), the side effects of suppression of TGF- $\beta 1$-induced metabolism of articular cartilage should be considered.

\section{Acknowledgements}

The present study was supported by the National Natural Science Foundation of China (grant no. 81101380).

\section{References}

1. Tchetina EV, Antoniou J, Tanzer M, Zukor DJ and Poole AR: Transforming growth factor-beta2 suppresses collagen cleavage in cultured human osteoarthritic cartilage, reduces expression of genes associated with chondrocyte hypertrophy and degradation, and increases prostaglandin E(2) production. Am J Pathol 168: 131-140, 2006

2. Ulrich-Vinther M, Stengaard C, Schwarz EM, Goldring MB and Soballe K: Adeno-associated vector mediated gene transfer of transforming growth factor-betal to normal and osteoarthritic human chondrocytes stimulates cartilage anabolism. Eur Cell Mater 10: 40-50, 2005.
3. Acosta CA, Izal I, Ripalda P, Douglas-Price AL and Forriol F: Gene expression and proliferation analysis in young, aged, and osteoarthritic sheep chondrocytes effect of growth factor treatment. J Orthop Res 24: 2087-2094, 2006.

4. Huang CY, Hagar KL, Frost LE, Sun Y and Cheung HS: Effects of cyclic compressive loading on chondrogenesis of rabbit bone-marrow derived mesenchymal stem cells. Stem Cells 22: 313-323, 2004.

5. Morigele M, Shao Z and Zhang Z, Kaige M, Zhang Y, Qiang W and Yang S: TGF- $\beta 1$ induces a nucleus pulposus-like phenotype in Notch 1 knockdown rabbit bone marrow mesenchymal stem cells. Cell Biol Int 37: 820-825, 2013.

6. Kwon YJ, Lee JW, Moon EJ, Chung YG, Kim OS and Kim HJ: Anabolic effects of Peniel 2000, a peptide that regulates TGF- $\beta 1$ signaling on intervertebral disc degeneration. Spine 38: E49-E58, 2013.

7. Roman-Blas JA, Stokes DG and Jimenez SA: Modulation of TGF-beta signaling by proinflammatory cytokines in articular chondrocytes. Osteoarthritis Cartilage 15: 1367-1377, 2007.

8. Qureshi HY, Sylvester J, El Mabrouk M and Zafarullah M: TGF-beta-induced expression of tissue inhibitor of metalloproteinases-3 gene in chondrocytes is mediated by extracellular signal-regulated kinase pathway and Sp1 transcription factor. J Cell Physiol 203: 345-352, 2005.

9. Yonekura A, Osaki M, Hirota Y, Tsukazaki T, Miyazaki Y, Matsumoto T, Ohtsuru A, Namba H, Shindo H and Yamashita S: Transforming growth factor-beta stimulates articular chondrocyte cell growth through p44/42 MAP kinase (ERK) activation. Endocr J 46: 545-553, 1999.

10. Re'em T, Kaminer-Israeli Y, Ruvinov $\mathrm{E}$ and Cohen S: Chondrogenesis of hMSC in affinity-bound TGF-beta scaffolds. Biomaterials 33: 751-761, 2012.

11. Qureshi HY, Ricci G and Zafarullah M: Smad signaling pathway is a pivotal component of tissue inhibitor of metalloproteinases-3 regulation by transforming growth factor beta in human chondrocytes. Biochim Biophys Acta 1783: 1605-1612, 2008.

12. Wang X, Li F, Fan C, Wang C and Ruan H: Effects and relationship of ERK1 and ERK2 in interleukin-1 $\beta$-induced alterations in MMP3, MMP13, type II collagen and aggrecan expression in human chondrocytes. Int J Mol Med 27: 583-589, 2011.

13. Schmittgen TD and Livak KJ: Analyzing real-time PCR data by the comparative C(T) method. Nat Protoc 3: 1101-1108, 2008.

14. Shi S, Mercer S, Eckert GJ and Trippel SB: Regulation of articular chondrocyte aggrecan and collagen gene expression by multiple growth factor gene transfer. J Orthop Res 30: 1026-1031, 2012.

15. Wrana JL, Attisano L, Wieser R, Ventura F and Massagué J: Mechanism of activation of the TGF-beta receptor. Nature 370: 341-347, 1994.

16. Heldin $\mathrm{CH}$, Miyazono $\mathrm{K}$ and ten Dijke P: TGF-beta signalling from cell membrane to nucleus through SMAD proteins. Nature 390: 465-471, 1997.

17. Shi Y and Massagué J: Mechanisms of TGF-beta signaling from cell membrane to the nucleus. Cell 113: 685-700, 2003.

18. Itoh S, Itoh F, Goumans MJ and Ten Dijke P: Signaling of transforming growth factor-beta family members through Smad proteins. Eur J Biochem 267: 6954-6967, 2000.

19. Massagué J and Wotton D: Transcriptional control by the TGF-beta/Smad signaling system. EMBO J 19: 1745-1754, 2000.

20. Cailotto F, Bianchi A, Sebillaud S, Venkatesan N, Moulin D, Jouzeau JY and Netter P: Inorganic pyrophosphate generation by transforming growth factor-beta-1 is mainly dependent on ANK induction by Ras/Raf-1/extracellular signal-regulated kinase pathways in chondrocytes. Arthritis Res Ther 9: R122, 2007.

21. O'Rear L, Longobardi L, Torello M, Law BK, Moses HL, Chiarelli F and Spagnoli A: Signaling cross-talk between IGF-binding protein-3 and transforming growth factor-(beta) in mesenchymal chondroprogenitor cell growth. J Mol Endocrinol 34: 723-737, 2005.

22. Shikhman AR, Brinson DC and Lotz MK: Distinct pathways regulate facilitated glucose transport in human articular chondrocytes during anabolic and catabolic responses. Am J Physiol Endocrinol Metab 286: E980-E985, 2004.

23. Miyazaki Y, Tsukazaki T, Hirota Y, Yonekura A, Osaki M, Shindo $\mathrm{H}$ and Yamashita S: Dexamethasone inhibition of TGF beta-induced cell growth and type II collagen mRNA expression through ERK-integrated AP-1 activity in cultured rat articular chondrocytes. Osteoarthritis Cartilage 8: 378-385, 2000.

24. Schneiderbauer MM, Dutton CM and Scully SP: Signaling "cross-talk" between TGF-beta1 and ECM signals in chondrocytic cells. Cell Signal 16: 1133-1140, 2004. 
25. Zhang M, Zhou Q, Liang QQ, Li CG, Holz JD, Tang D, Sheu TJ, $\mathrm{Li} \mathrm{TF}$, Shi Q and Wang YJ: IGF-1 regulation of type II collagen and MMP-13 expression in rat endplate chondrocytes via distinct signaling pathways. Osteoarthritis Cartilage 17: 100-106, 2009.

26. Park MS, Kim YH, and Lee JW: FAK mediates signal crosstalk between type II collagen and TGF-beta 1 cascades in chondrocytic cells. Matrix Biol 29: 135-142, 2010.

27. Kimura H, Yukitake H, Suzuki H, Tajima Y, Gomaibashi K, Morimoto S, Funabashi Y, Yamada K and Takizawa M: The chondroprotective agent ITZ-1 inhibits interleukin-1beta-induced matrix metalloproteinase-13 production and suppresses nitric oxide-induced chondrocyte death. J Pharmacol Sci 110: 201-211, 2009.
28. Megías J, Guillén MI, Bru A, Gomar F and Alcaraz MJ: The carbon monoxide-releasing molecule tricarbonyldichlororuth enium(II) dimer protects human osteoarthritic chondrocytes and cartilage from the catabolic actions of interleukin-1beta. J Pharmacol Exp Ther 325: 56-61, 2008.

29. Iwayama H, Sakamoto T, Nawa A and Ueda N: Crosstalk between Smad and Mitogen-Activated Protein Kinases for the Regulation of Apoptosis in Cyclosporine A- Induced Renal Tubular Injury. Nephron Extra 1: 178-189, 2011.

30. Javelaud D and Mauviel A: Crosstalk mechanisms between the mitogen-activated protein kinase pathways and Smad signaling downstream of TGF-beta: Implications for carcinogenesis. Oncogene 24: 5742-5750, 2005 